\title{
Microparticles and a P-selectin-mediated pathway of blood coagulation
}

\author{
Alessandro Celi ${ }^{\mathrm{a}, \mathrm{c}}$, Roberto Lorenzet ${ }^{\mathrm{b}}$, Barbara C. Furie ${ }^{\mathrm{c}}$ and Bruce Furie ${ }^{\mathrm{c}, *}$ \\ ${ }^{a}$ Laboratorio di Biologia Cellulare Respiratoria, Dipartimento Cardiotoracico dell'Università di Pisa, Pisa, Italy \\ ${ }^{\mathrm{b}}$ Antonio Taticchi Unit for Atherosclerosis and Thrombosis, Istituto Ricerche Farmacologiche Mario Negri, \\ Consorzio Mario Negri Sud, S. Maria Imbaro, Italy \\ ${ }^{\mathrm{c}}$ Center for Hemostasis and Thrombosis Research, Beth Israel Deaconess Medical Center and Harvard Medical \\ School, Boston, MA, USA
}

\section{Introduction}

A classical model that describes blood coagulation as a multi-step series of events was developed nearly 40 years ago $[1,2]$ and, while being constantly refined and ameliorated, has remained mostly valid to date. The current model of this "coagulation cascade" describes two distinct pathways, both eventually leading to the activation of prothrombin to thrombin which in turn is responsible for fibrin formation. The intrinsic pathway is initiated by the activation of Factor XII to Factor XIIa, the enzyme form of the zymogen Factor XII. The extrinsic pathway, considered the physiologic initiator of blood coagulation, is triggered by the formation of a complex that includes Factor VIIa, tissue factor, phospholipid membranes and calcium ions. Anionic phospholipids, mainly phosphatidylserine, are critical components of this complex and it is commonly accepted that activated platelets, in the absence of primary in vivo data, accumulate at the site of tissue injury and provide most of these negatively charged species. The activation of this pathway catalyzes the conversion of Factor IX and Factor X to Factor IXa and Factor Xa, respectively [3].

${ }^{*}$ Corresponding author: Dr. Bruce Furie, Center for Hemostasis and Thrombosis Research, Research East 319, Beth Israel Deaconess Medical Center, 330 Brookline Ave., Boston MA 02115, USA. Tel.: +1 617667 0620; Fax: +1 617975 5505; E-mail: bfurie@ caregroup.harvard.edu.
Tissue factor is an integral membrane protein with a molecular weight of 47,000 constitutively expressed by non vascular cells, and therefore extrinsic to blood [4]. According to the accepted model, a small amount of Factor VII circulates in its active form as Factor VIIa but is kept physically segregated from tissue factor by the integrity of the endothelium and has no significant biologic activity. Upon vascular damage Factor VIIa comes in contact with tissue factor expressed by the underlying non-vascular tissues when these tissues are exposed to flowing blood, and Factor IXa and Factor $\mathrm{Xa}$ are generated. This model predicts that tissue factor is not constitutively synthesized by cells normally in contact with the blood stream, lest intravascular coagulation ensues. However, endothelial cells and monocytes can synthesize tissue factor upon activation [5, 6]. Agonists classically recognized to cause synthesis of tissue factor by endothelial cells and/or monocytes include bacter bacterial endotoxin (lipopolysaccharide, LPS), Interleukin-1 (IL-1), Tumor Necrosis Factor- $\alpha(\mathrm{TNF}-\alpha)$, immune complexes, platelets and platelet membranes. However, the list is constantly growing [7-9]. Whether other types of white cells beside the monocyte synthesize and express tissue factor upon activation is still a matter of debate [8,10-13]. It must be emphasized that tissue factor expression by leukocytes and endothelial cells involves de novo protein synthesis and requires several hours. Monocyte and endothelial cell-derived tissue factor is therefore unlikely to be involved in triggering blood coagulation 
upon tissue injury but rather has been mostly linked to wound repair and remodeling, thrombus formation and atherosclerosis [14]. Finally, tissue factor has also been demonstrated in plasma in minute concentrations of about 100-150 ng/ml [15-17]. The physiologic relevance of circulating tissue factor has remained obscure.

Over the past several years, experimental data have been obtained that do not fit this classical model. In 1984 we [18] and others [19] independently discovered a protein expressed on the platelet surface only upon activation. The protein was eventually shown to be a vascular cell adhesion molecule that mediated leukocyte-platelet and endothelial cell-platelet interaction, and was renamed P-selectin [20]. The presence of P-selectin was also demonstrated in endothelial cells $[21,22]$. While the first evidence that P-selectin was indeed an adhesion molecule was obtained in vitro, the function of the protein was subsequently investigated in an animal model [23]. An artero-venous shunt implanted in a baboon (Papio Cynocephalus) was fitted with a Dacron graft. Under the experimental conditions used the graft is highly thrombogenic and a thrombus formed rapidly. When autologous radiolabelled leukocytes were injected they rapidly accumulated in the graft, as expected. Antibodies to P-selectin inhibited leukocyte accumulation, thus confirming the role of P-selectin as a platelet-leukocyte adhesion molecule in an in vivo animal model. However, it was also observed that beside inhibiting leukocyte accumulation, antibodies to P-selectin were also able to inhibit fibrin deposition within the grafts. This observation was totally unexpected and could not be explained based on the current knowledge of blood coagulation that did not propose a role for P-selectin. While monocyte accumulation was likely inhibited by P-selectin blockade, this could not account for fibrin inhibition since these cells should not represent a significant source of tissue factor in the short time frame, measured in minutes, of our model. This work documented a P-selectin-dependent pathway of blood coagulation and demonstrated for the first time a link between P-selectin and fibrin formation, but the molecular basis of this observation was obscure.

To investigate the possible role of P-selectin in fibrin formation, we tested the hypothesis that binding of monocytes to P-selectin expressed by activated platelets within the thrombus upregulates tissue factor activity. Platelets and platelet membranes had long been included in the list of agonists capable of monocyte activation. Freshly isolated peripheral blood monocytes cultured in the presence of soluble P-selectin or coin- cubated with Chinese hamster ovary $(\mathrm{CHO})$ cells transfected with the cDNA for P-selectin expressed procoagulant activity (as assessed by a clotting assay), tissue factor antigen (as assessed by immunofluorescence) and tissue factor mRNA (as assessed by semiquantitative RT-PCR). The effect was inhibited by a monoclonal antibody to P-selectin [24]. This was the first demonstration that P-selectin, beside its role as a docking molecule important for the initial steps of the inflammatory response, had also a signaling function involved in the activation of blood coagulation. However, tissue factor expression by P-selectin-activated monocytes required protein synthesis and the time scale of fibrin formation could not explain the results obtained in the baboon model.

In experiments aimed at investigating the role of circulating tissue factor in blood coagulation, Giesen and coworkers employed an in vitro system in which collagen coated glass slides or pig arterial media were exposed to flowing human blood in a perfusion parallel flow chamber [12]. While neither surface contained human tissue factor at the beginning of the experiment, microscopic analysis of the thrombus showed the formation of tissue factor-rich microthrombi after a 5 minute perfusion with blood directly flowing from a normal volunteer's vein. The appearance of tissue factor was well before any de novo synthesis of tissue factor by monocytes might occur. Although tissue factorcontaining leukocytes were detected by immunostaining, much of the protein appeared to be extracellular. Under the experimental conditions described, the flowing blood is the only possible source of tissue factor. These observations led the authors to conclude that blood-borne TF is functionally active and potentially thrombogenic.

In order to gain a better understanding of the complex molecular mechanisms of blood coagulation in vivo, we have recently developed an intravital microscopy system for real-time imaging of thrombus formation in mice. The system has been described in detail elsewhere $[25,26]$. Briefly, the cremaster muscle of a living mouse is exteriorized and pinned across a coverslip mounted on a custom-designed tray. This allows direct visualization of the microcirculation of the living animal. The preparation is then placed on the stage of a fluorescence microscope that includes both widefield and confocal capabilities. To induce thrombus formation, laser pulses, focused through the microscope lenses, injure the vascular wall of either arterioles or venules. Fluorescently labeled cells, or antibodies to circulating proteins or cell surface antigens are injected through the 
jugular vein to visualize single thrombus components. Data from up to three different fluorescent channels plus brightfield can be digitally recorded for off-line analysis. The confocal component of the microscope employs Nipkow disk technology (as opposed to the point scanning technique used by traditional confocal microscopes) to collect images at video frame rates.

We took advantage of this instrumentation to revisit the role of $\mathrm{P}$-selectin in fibrin deposition. Genetically engineered mice lacking the leukocyte receptor for P-selectin, P-Selectin Glycoprotein Ligand-1 (PSGL-1), have been generated in our laboratory [27]. By injecting fluorescently labeled antibodies to fibrin we were able to demonstrate that fibrin deposition in PSGL-1 $1^{-/-}$mice is impaired compared to wild type mice. Similar results were obtained with $\mathrm{P}$-selectin ${ }^{-/-}$ mice and in wild-type mice treated with blocking antibodies to P-selectin [28]. These results confirm and extend those obtained in the baboon model over a decade earlier and again support the existence of a Pselectin/PSGL-1-dependent pathway of blood coagulation. To investigate whether this pathway involves tissue factor, fluorescently labeled anti-tissue factor antibodies were also injected to detect tissue factor in the developing thrombus. Tissue factor antigen accumulated at the thrombus/vessel wall interface as well as within the developing thrombus [28]. Tissue factor accumulation in the thrombus was indeed impaired in PSGL-1 $1^{-/-}$and P-selectin ${ }^{-/}$mice. These data indicate that the P-selectin/PSGL-1 receptor pair is required for tissue factor accumulation at sites of vessel wall injury.

It has been known for over fifteen years that many eukaryotic cells release vesicles either spontaneously or upon stimulation. Some of these vesicles originate directly from the cell membrane and have been given different names, including ectosomes [29], microvesicles [30], and micoparticles [31]. Platelets, for example, express microparticles upon stimulation [32]; these microparticles can be detected in the peripheral blood by flow cytometry and their number correlates with the extent of platelet activation [31]. An incomplete list of biological functions of microparticles reported in vitro includes the presence of binding sites for Factor VIII in platelet-derived microparticles [33], upregulation of endothelial cell synthesis of proinflammatory cytokines by neutrophil-derived microparticles [34], transfer of the chemokine receptor CCR5 from monocytes to cells not expressing it, thus allowing HIV infection of the latter [35]. More relevant to the purpose of this review, the formation of pro- coagulant microparticles by monocytes has also been demonstrated [36]. Monocytes exposed to LPS release prothrombinase activity in the supernatant that is likely attributable to phosphatidylserine-expressing vesicles. This was confirmed by flow cytometry, probing the cell-free supernatant of LPS stimulated monocytes with fluorescein-labeled annexin V. In addition, these microparticles express tissue factor activity, as assessed by a Factor Xa generation assay. Finally, flow cytometry also shows that antibodies directed against several cell surface antigens, including CD14, CD11a, and CD18, bind to microparticles. Taken together these results indicate that upon LPS stimulation, monocytes shed microparticles that are potentially responsible for the dissemination of procoagulant activity; these microparticles also express at least some of the integral membrane proteins expressed by intact monocytes. While one might be tempted to consider microparticles as in vitro artifacts or, possibly, cell debris devoid of functional significance in vivo, several authors have demonstrated increased numbers of microparticles originating from cells of different lineage in a variety of diseases suggesting that their formation is a regulated process with physiologic implications [37-44].

We have analyzed freshly isolated cell-free plasma from normal volunteers for the presence of microparticles bearing both tissue factor and PSGL-1. Polystyrene beads coated with anti-tissue factor antibodies were incubated with cell-free plasma to capture tissue factor-bearing microparticles. The beads were then sedimented by centrifugation, incubated with fluorescein labeled anti PSGL-1 antibodies and analyzed by flow-cytometry [28]. By this method we were able to demonstrate the presence in unstimulated human blood of circulating microparticles that are captured by anti-tissue factor antibodies and stain with anti PSGL-1 antibodies, and therefore must express both antigens. Using the parallel flow chamber described above, transfer of tissue factor-bearing microparticles from leukocytes to platelets adherent to the collagen coated slide has been suggested based on immunoelectron microscopy [45]. Colocalization on these particles of tissue factor and CD15, a carbohydrate moiety that is a component of numerous leukocyte membrane proteins including PSGL-1, and is part of the recognition site of this protein for P-selectin [46], was observed.

This tissue factor-initiated P-selectin/PSGL-1mediated blood coagulation pathway appears to play a role in thrombogenesis. This pathway is distinct from the classical pathway in which extrinsic tissue factor in non-vascular cells becomes exposed to flowing blood, 
and thus initiates blood coagulation directly. In this second pathway, monocytes, constitutively expressing PSGL-1, are activated by as yet unknown agonists in vivo to synthesize and express tissue factor on their surface. The same agonists or other molecules then signal the cells to shed microparticles expressing both tissue factor and PSGL-1 on their surface. These microparticles circulate at a relatively low concentration, and tissue factor is either inactive or too dilute to initiate blood coagulation. Upon vessel wall injury, platelets accumulate. Upon their activation, they express P-selectin. The circulating PSGL-1-bearing microparticles bind to the bound activated platelets, delivering tissue factor to the developing platelet thrombus. Activated platelets on the surface of the Dacron shunt or at the site of laserinduced vessel wall injury capture tissue factor [23, 28]. Once concentrated within the thrombus, tissue factor reaches a critical concentration and triggers the initiation of blood coagulation.

While the presence and functional significance of circulating tissue factor is gaining attention, tissue factor constitutively expressed by non-vascular cells is clearly important for normal hemostasis. With the availability of the intravascular microscopy system described above, we set out to compare the role of both extrinsic and blood-borne tissue factor in blood coagulation. Genetically engineered mice expressing very low level of TF (approximately 1\% compared to wild type) have been generated. These "low TF" mice, unlike $\mathrm{TF}^{-/-}$mice that die during embryonic development, show normal growth and development [47]. We transplanted bone marrow obtained from low TF mice into wild type mice and bone marrow from wild type mice into low TF mice. This approach allowed us to distinguish the relative contribution of tissue factor generated by hematopoietic cells from that of non-hematopoietic cell-derived tissue factor. Platelet, fibrin and tissue factor accumulation within the thrombus were analyzed in real time upon laser induced tissue injury. Low TF mice generated platelet thrombi that were markedly reduced in size and that lacked both tissue and fibrin. These results indicate an important role for tissue factor-initiated thrombin generation in platelet activation. Chimeric mice expressing normal vessel wall tissue factor but minimal hematopoietic cell-derived tissue factor displayed a modest reduction in platelet thrombus size but markedly impaired tissue factor and fibrin deposition within the thrombus. On the contrary, laser-induced vessel injury in chimeric mice expressing normal bone marrow-derived tissue factor but low vessel wall tissue factor yielded a modest reduction in platelet thrombus size but had normal tissue factor and fibrin content [48]. These data allowed us to conclude that hematopoietic and tissue derived tissue factor play distinct roles in blood coagulation and thrombogenesis. Extrinsic tissue factor acts rapidly upon tissue injury to generate thrombin that activates platelets adherent to the vessel wall;presumably these platelets release ADP, which in turns activates other platelets, leading to the formation and rapid growth of a platelet thrombus. Tissue factor derived from hematopoietic cells, blood borne tissue factor, then leads to the generation of fibrin within the growing thrombus. Since we have shown that this phenomenon requires P-selectin/PSGL-1 interaction, we maintain that blood borne tissue factor is associated with PSGL-1-bearing microparticles.

The agonist(s) that signal leukocytes to express tissue factor and shed tissue factor/PSGL-1 bearing microparticles have not been identified. Andre and coworkers have engineered a mouse strain that expresses a truncated form of P-selectin lacking the cytoplasmic tail [49]. These mice have an approximately threefold increase in the concentration of circulating soluble P-selectin which results in a procoagulant state. These mice have a higher level of circulating microparticles expressing tissue factor. These microparticles also express the integrin $\alpha_{M} \beta_{2}$ (Mac-1), suggesting their leukocytic origin. Furthermore, incubation of human blood with a P-selectin-Ig chimera shortens the whole blood clotting time whereas mice deficient in PSGL-1 have lower numbers of microparticles than wild type mice [50]. It would appear that P-selectin may be involved in the generation of tissue factor- bearing microparticles by leukocytes but the presence of microparticles in PSGL-1 null and P-selectin null mice indicates that multiple mechanisms beside P-selectin and PSGL-1 are important in microparticle generation.

Many questions remain to be answered. Firstly, the methods used to quantitate microparticles and associated antigens in blood or platelet-poor plasma by ourselves and others are inadequate. These measurements are based upon light scattering during flow cytometry, and light scattering of small particles is non-linear with regard to particle size and inaccurate. Microparticles, cellular debris and instrument noise are not easily separated by flow cytometry. Furthermore, formal quantitation of tissue factor antigen on microparticles, and not simply microparticle concentration, is important since most microparticles in blood are not derived from leukocytes and may have little or nothing to do with thrombogenesis. Second, the functional state of tissue factor associated with blood-borne microparticles is 
unknown since efforts are usually directed to assaying tissue factor antigen. Is blood-borne tissue factor functionally active? What are the signals and the molecular basis for its tissue factor activation? Third, are there proteins uniquely expressed on the microparticle that are not expressed on the parent cell from which the microparticle is derived? Fourth, what is the mechanism of microparticle generation and what is the fate of circulating microparticles? With our demonstration of the incorporation of leukocyte-derived microparticles into the developing thrombus in an interaction mediated by P-selectin and PSGL-1, we provide the first insight into a physiologic role for microparticles in their contribution to the development of fibrin during thrombus formation in a living animal. Further developments in the technology of microparticle analysis and their application to study of tissue factor initiation of blood coagulation should prove fruitful in future investigations.

\section{References}

[1] E. Davie and O. Ratnoff, Waterfall sequence for intrinsic blood cotting, Science 145 (1964), 1310-1312.

[2] R. Macfarlane, An enzyme cascade in the blood clotting mechanism, and its function as a biochemical amplifier, Nature 202 (1964), 498-499.

[3] B. Furie and B. Furie, The molecular basis of blood coagulation, Cell 53 (1988), 505-518.

[4] T. Drake, J. Morrissey and T. Edgington, Selective cellular expression of tissue factor in human tissues. Implications for disorders of hemostasis and thrombosis, Am J Pathol 134 (1989), 1087-1097.

[5] N. Semeraro, A. Biondi, R. Lorenzet, D. Locati, A. Mantovani and M. Donati, Direct induction of tissue factor synthesis by endotoxin in human macrophages from diverse anatomical sites, Immunology 50 (1983), 529-535.

[6] M. Colucci, G. Balconi, R. Lorenzet et al., Cultured human endothelial cells generate tissue factor in response to endotoxin, J Clin Invest 71 (1983), 1893-1896.

[7] E. Camerer, A. Kolsto and H. Prydz, Cell biology of tissue factor, the principal initiator of blood coagulation, Thromb Res 81 (1996), 1-41.

[8] B. Osterud, Tissue factor expression by monocytes: regulation and pathophysiological roles, Blood Coagul Fibrinolysis 9(1) (1998), S9-S14.

[9] R. Lorenzet, E. Napoleone, A. Celi, G. Pellegrini and A. Di Santo, Cell-cell interaction and tissue factor expression, Blood Coagul Fibrinolysis 9(1) (1998), S49-S59.

[10] A. Higure, K. Okamoto, K. Hirata et al., Macrophages and neutrophils infiltrating into the liver are responsible for tissue factor expression in a rabbit model of acute obstructive cholangitis, Thromb Haemost 75 (1996), 791-795.

[11] H. Todoroki, S. Nakamura, A. Higure et al., Neutrophils express tissue factor in a monkey model of sepsis, Surgery $\mathbf{1 2 7}$ (2000), 209-216.

[12] P.L.A. Giesen, U. Rauch, B. Bohrmann et al., Blood-borne tissue factor: Another view of thrombosis, PNAS 96 (1999), 2311-2315.
[13] B. Bouchard and P. Tracy, Platelets, leukocytes, and coagulation, Curr Opin Hematol 8 (2001), 263-269.

[14] U. Pendurthi and L. Rao, Factor VIIa/tissue factor-induced signaling: a link between clotting and disease, Vitam Horm 64 (2002), 323-355.

[15] T. Koyama, K. Nishida, S. Ohdama et al., Determination of plasma tissue factor antigen and its clinical significance, $\mathrm{Br} \mathrm{J}$ Haematol 87 (1994), 343-347.

[16] M. Zumbach, M. Hofmann, V. Borcea et al., Tissue factor antigen is elevated in patients with microvascular complications of diabetes mellitus, Exp Clin Endocrinol Diabetes 105 (1997), 206-212.

[17] J. Fareed, D. Callas, D. Hoppensteadt and E.J. Bermes, Tissue factor antigen levels in various biological fluids, Blood Coagul Fibrinolysis 6 (1995), S32-S36.

[18] S. Hsu-Lin, C. Berman, B. Furie, D. August and B. Furie, A platelet membrane protein expressed during platelet activation and secretion. Studies using a monoclonal antibody specific for thrombin- activated platelets, J. Biol. Chem. 259 (1984), 9121-9126.

[19] R. McEver and M. Martin, A monoclonal antibody to a membrane glycoprotein binds only to activated platelets, J. Biol. Chem. 259 (1984), 9799-9804.

[20] E. Larsen, A. Celi, G. Gilbert et al., PADGEM protein: a receptor that mediates the interaction of activated platelets with neutrophils and monocytes, Cell 59 (1989), 305-312.

[21] R. Bonfanti, BC F, B F and D. Wagner, PADGEM (GMP140) is a component of Weibel-Palade bodies of human endothelial cells, Blood 73 (1989), 1109-1112.

[22] R. McEver, J. Beckstead, K. Moore, L. Marshall-Carlson and D. Bainton, GMP-140, a platelet alpha-granule membrane protein, is also synthesized by vascular endothelial cells and is localized in Weibel-Palade bodies, J Clin Invest 84 (1989), 92-99.

[23] T. Palabrica, R. Lobb, BC F et al., Leukocyte accumulation promoting fibrin deposition is mediated in vivo by P-selectin on adherent platelets, Nature 359 (1992), 848-851.

[24] A. Celi, G. Pellegrini, R. Lorenzet et al., P-Selectin Induces the Expression of Tissue Factor on Monocytes, PNAS 91 (1994), 8767-8771.

[25] S. Falati, P. Gross, G. Merrill-Skoloff, B. Furie and B. Furie, Real-time in vivo imaging of platelets, tissue factor and fibrin during arterial thrombus formation in the mouse, Nat. Med. 8 (2002), 1175-1181.

[26] A. Celi, G. Merrill-Skoloff, P. Gross et al., Thrombus formation: direct real-time observation and digital analysis of thrombus assembly in a living mouse by confocal and widefield intravital microscopy, J Thromb Haemost 1 (2003), 6068.

[27] J. Yang, T. Hirata, K. Croce et al., Targeted Gene Disruption Demonstrates That P-Selectin Glycoprotein Ligand 1 (PSGL1) Is Required for P-Selectin-mediated but Not E-Selectinmediated Neutrophil Rolling and Migration, J. Exp. Med. 190 (1999), 1769-1782.

[28] S. Falati, Q. Liu, P. Gross et al., Accumulation of Tissue Factor into Developing Thrombi In Vivo Is Dependent upon Microparticle P-Selectin Glycoprotein Ligand 1 and Platelet P-Selectin, J. Exp. Med. 197 (2003), 1585-1598.

[29] C. Hess, S. Sadallah, A. Hefti, R. Landmann and J-A. Schifferli, Ectosomes Released by Human Neutrophils Are Specialized Functional Units, J Immunol 163 (1999), 4564-4573.

[30] A. MacKenzie, H. Wilson, E. Kiss-Toth, S. Dower, R. North and A. Surprenant, Rapid secretion of interleukin-1beta by microvesicle shedding, Immunity 15 (2001), 825-835. 
[31] C. Abrams, N. Ellison, A. Budzynski and S. Shattil, Direct detection of activated platelets and platelet-derived microparticles in humans, Blood 75 (1990), 128-138.

[32] P. Sims and T. Wiedmer, Repolarization of the membrane potential of blood platelets after complement damage: evidence for a $\mathrm{Ca}++-$-dependent exocytotic elimination of $\mathrm{C} 5 \mathrm{~b}-9$ pores, Blood 68 (1986), 556-561.

[33] G. Gilbert, P. Sims, T. Wiedmer, B. Furie, B. Furie and S. Shattil, Platelet-derived microparticles express high affinity receptors for factor VIII, J. Biol. Chem. 266 (1991), 1726117268.

[34] M. Mesri and D.C. Altieri, Endothelial Cell Activation by Leukocyte Microparticles, J Immunol 161 (1998), 4382-4387.

[35] M. Mack, A. Kleinschmidt, H. Bruhl et al., Transfer of the chemokine receptor CCR5 between cells by membranederived microparticles: A mechanism for cellular human immunodeficiency virus 1 infection, Nat Med 6 (2000), 769-775.

[36] N. Satta, F. Toti, O. Feugeas et al., Monocyte vesiculation is a possible mechanism for dissemination of membraneassociated procoagulant activities and adhesion molecules after stimulation by lipopolysaccharide, J Immunol 153 (1994), 3245-3255.

[37] V. Gonzalez-Quintero, J. Jimenez, W. Jy et al., Elevated plasma endothelial microparticles in preeclampsia, Am J Obstet Gynecol 189 (2003), 589-593.

[38] K. Aupeix, B. Hugel, T. Martin et al., The Significance of Shed Membrane Particles during Programmed Cell Death In Vitro, and In Vivo, in HIV-1 Infection, J. Clin. Invest. 99 (1997), 1546-1554.

[39] K. Joop, R. Berckmans, R. Nieuwland et al., Microparticles from patients with multiple organ dysfunction syndrome and sepsis support coagulation through multiple mechanisms, Thromb Haemost 85 (2001), 810-820.

[40] Z. Mallat, B. Hugel, J. Ohan, G. Leseche, J.-M. Freyssinet and A. Tedgui, Shed Membrane Microparticles With Procoagulant Potential in Human Atherosclerotic Plaques: A Role for Apoptosis in Plaque Thrombogenicity, Circulation 99 (1999), 348-353.
[41] Z. Mallat, H. Benamer, B. Hugel et al., Elevated Levels of Shed Membrane Microparticles With Procoagulant Potential in the Peripheral Circulating Blood of Patients With Acute Coronary Syndromes, Circulation 101 (2000), 841-843.

[42] M. Nagahama, S. Nomura, S. Kanazawa, Y. Ozaki, H. Kagawa and S. Fukuhara, Significance of anti-oxidized LDL antibody and monocyte-derived microparticles in anti-phospholipid antibody syndrome, Autoimmunity 36 (2003), 125-131.

[43] R. Nieuwland, R.J. Berckmans, R.C. Rotteveel-Eijkman et al., Cell-Derived Microparticles Generated in Patients During Cardiopulmonary Bypass Are Highly Procoagulant, Circulation 96 (1997), 3534-3541.

[44] R. Nieuwland, R.J. Berckmans, S. McGregor et al., Cellular origin and procoagulant properties of microparticles in meningococcal sepsis, Blood 95 (2000), 930-935.

[45] U. Rauch, D. Bonderman, B. Bohrmann et al., Transfer of tissue factor from leukocytes to platelets is mediated by CD15 and tissue factor, Blood 96 (2000), 170-175.

[46] E. Larsen, T. Palabrica, S. Sajer et al., PADGEM-dependent adhesion of platelets to monocytes and neutrophils is mediated by a lineage-specific carbohydrate, LNF III (CD15), Cell 63 (1990), 467-474.

[47] R. Pawlinski, A. Fernandes, B. Kehrle et al., Tissue factor deficiency causes cardiac fibrosis and left ventricular dysfunction, PNAS 99 (2002), 15333-15338.

[48] J. Chou, N. Mackman, G. Merrill-Skoloff, B. Pedersen, B. Furie and B. Furie, Initiation and propagation of the arterial thrombus: contributions of vesel wall and blood-borne Tissue Factor, Blood (2004), in press.

[49] P. Andre, D. Hartwell, I. Hrachovinova, S. Saffaripour and D.D. Wagner, Pro-coagulant state resulting from high levels of soluble P-selectin in blood, PNAS 97 (2000), 13835-13840.

[50] I. Hrachovinova, B. Cambien, A. Hafezi-Moghadam et al., Interaction of P-selectin and PSGL-1 generates microparticles that correct hemostasis in a mouse model of hemophilia, $A$. Nat. Med. 9 (2003), 1020-1025. 


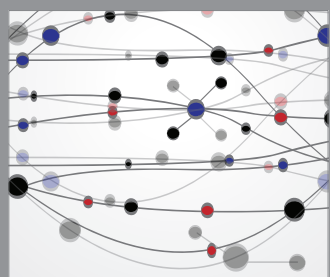

The Scientific World Journal
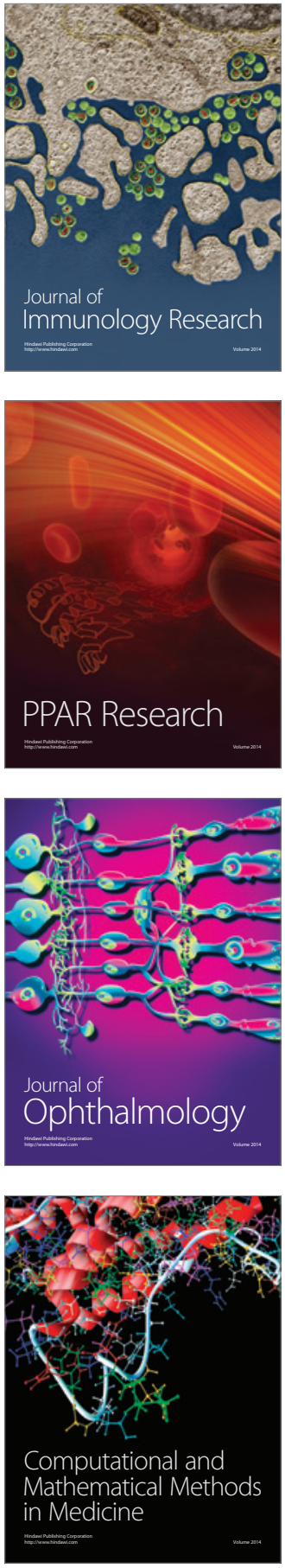

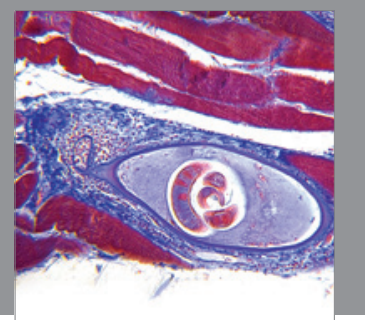

Gastroenterology

Research and Practice
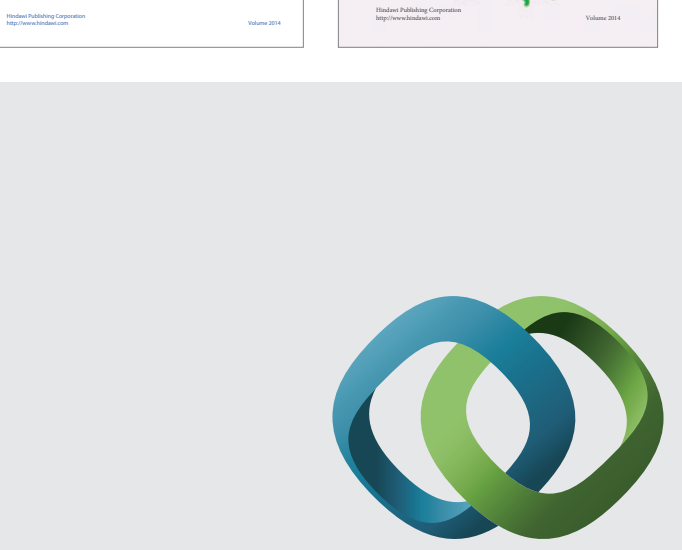

\section{Hindawi}

Submit your manuscripts at

http://www.hindawi.com
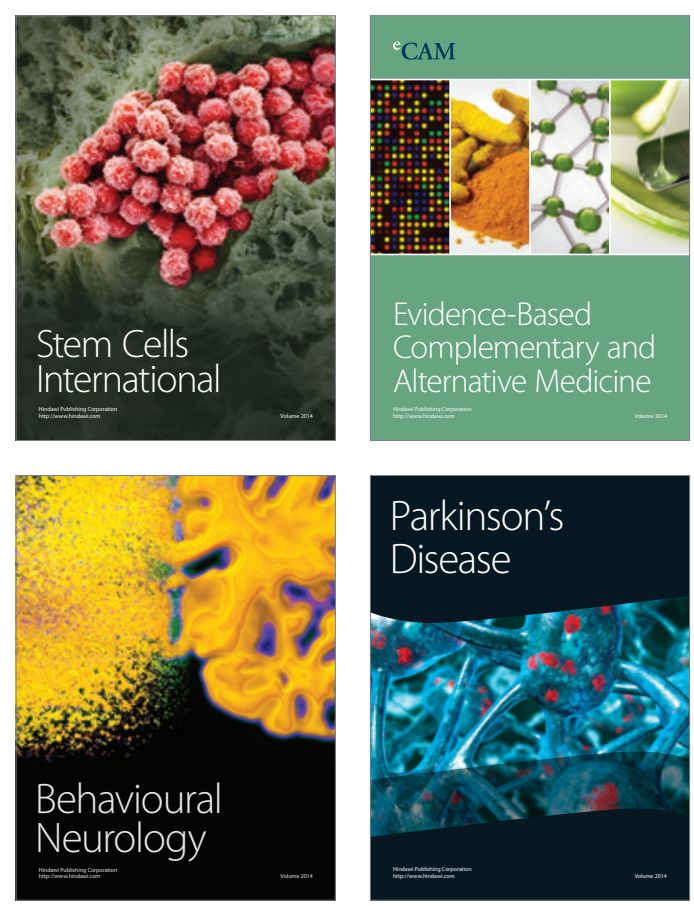

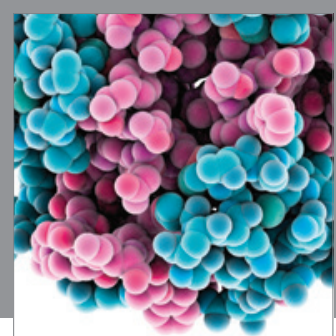

Journal of
Diabetes Research

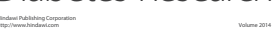

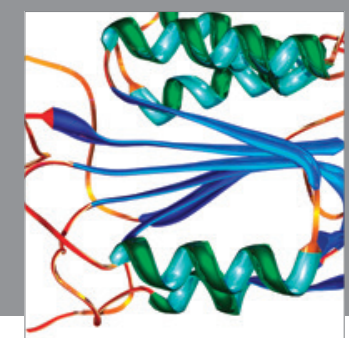

Disease Markers
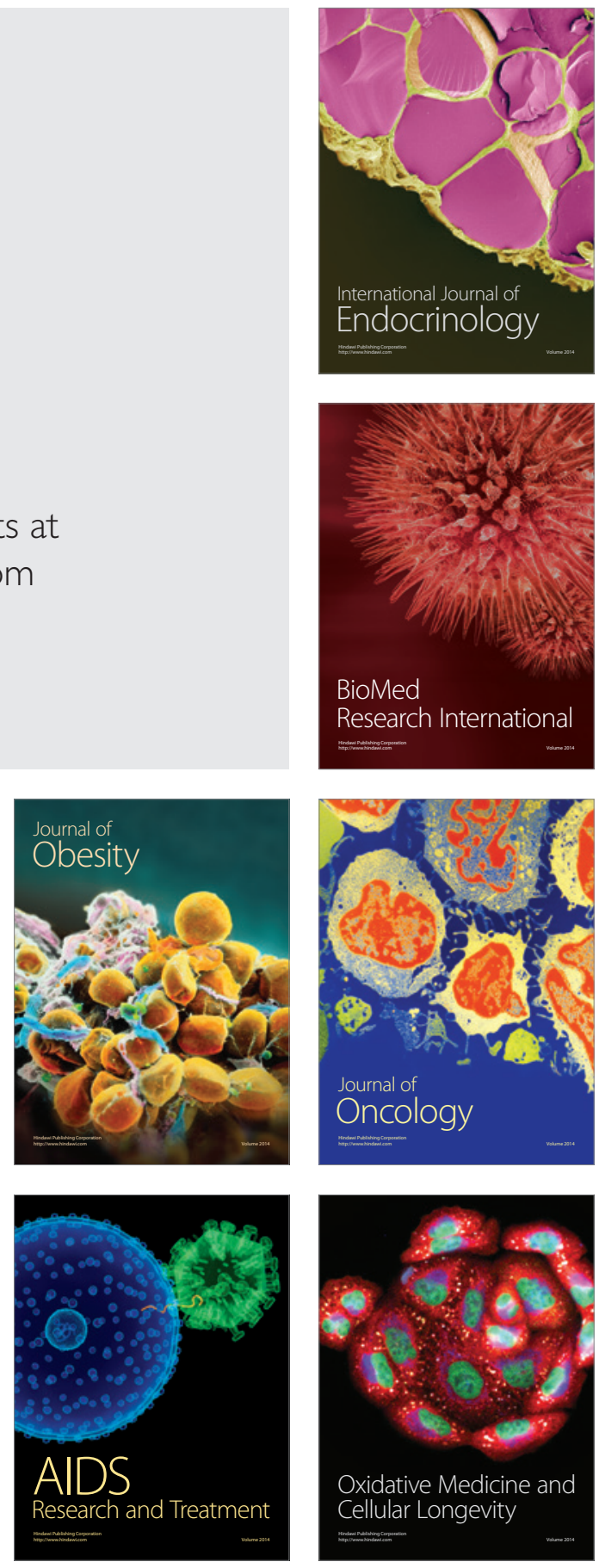